Michael A. Downing, BS, Michael O. Bazzi, BS, Mark E. Vinicky, MS, Nicholas V. Lampasona, BS, Oleg Tsvyetayev, BS and Harvey N. Mayrovitz*, PhD

\title{
Dietary views and habits of students in health professional vs. non-health professional graduate programs in a single university
}

https://doi.org/10.1515/jom-2020-0178

Received July 11, 2020; accepted August 21, 2020;

published online February 12, 2021

\section{Abstract}

Context: Students enrolled in health professional (HP) programs receive varying amounts of credit hours dedicated to nutritional education, and obesity remains an issue in the United States among healthcare providers.

Objectives: To assess whether HP students differ in nutrition and exercise habits from non-health professional (NHP) students at a single university, and whether any gender-related differences existed in those habits.

Methods: From September 25, 2018 to October 10, 2019, a 16-question multiple-choice survey was distributed via e-mail or in person to HP and NHP students enrolled at Nova Southeastern University (NSU) in Fort Lauderdale, Florida. Questions targeted participant dietary and exercise habits. Each question had five multiple-choice answer options, each of which was assigned a coded value to compare similarities and differences between the HP and NHP groups.

Results: Of 732 responses (569 HP, 163 NHP), results showed no statistically significant difference between enrollment groups $(\mathrm{p}>0.05)$ in any response parameter including consumption of sweets, fast food, red meat, caffeine, water, fruit, and vegetables. Comparisons among sexes demonstrated significant differences. Women consumed less red meat, water, and protein, and women

*Corresponding author: Harvey N. Mayrovitz, PhD, Dr. Kiran C. Patel College of Allopathic Medicine, Nova Southeastern University, 3200 S. University Drive, Davie, 33328-5326, Fort Lauderdale, FL, USA,

E-mail: mayrovit@nova.edu

Michael A. Downing, BS, Michael O. Bazzi, BS, Mark E. Vinicky, MS, Nicholas V. Lampasona, BS and Oleg Tsvyetayev, BS, Dr. Kiran C. Patel College of Osteopathic Medicine, Nova Southeastern University, Fort Lauderdale, FL, USA participated in less exercise compared to men. Women also consumed more sweets compared to men.

Conclusions: Results suggest that NSU students enrolled in HP and NHP programs have similar nutritional concepts and eating habits. This may indicate a need to strengthen nutritional education in dietary health and wellness for HP students.

Keywords: diet; graduate school; health professional; non-health professional; nutrition; obesity.

The incidence of obesity, defined as a body mass index (BMI) greater than $30 \mathrm{~kg} / \mathrm{m}^{2}$, has become an epidemic in the United States [1, 2]. A systematic review of controlled trials [3] showed that nutritional plans and physical activity leading to weight loss of $\geq 5 \%$ yielded improvements in patients' lipid panels, including triglycerides, total cholesterol, low-density lipoprotein (LDL), and highdensity lipoprotein (HDL) [3]. As of 2016, the Centers for Disease Control and Prevention (CDC) estimated that roughly $40 \%$ of adults in the United States were obese [4]. Meanwhile, obesity among college graduates was $22.7 \%$ in 2016 [4]. Even in the "healthiest" age demographic in the United States (adults aged 18-24), the prevalence of obesity is $16.5 \%$ [4], which is still higher than the worldwide prevalence of $13.5 \%$ [5].

Studies have also demonstrated increasing rates of obesity among healthcare providers. According to a study published in the American Journal of Preventative Medicine [6], US healthcare workers had a 22\% obesity prevalence in 2010. Obesity among nurses is especially elevated, according to a survey distributed to American nursing professionals, which revealed that $54 \%$ of the 760 respondents were overweight or obese (mean BMI, $27.2 \mathrm{~kg} /$ $\mathrm{m}^{2}$ ) [7]. Furthermore, certain studies have indicated that unhealthy habits practiced among healthcare providers may have originated during their time in health professional (HP) school [8, 9]. Unhealthy habits among medical students have been shown to impact their counseling of patients. For 
example, a "Healthy Doctor = Healthy Patient" survey [8] in 2015 among medical students at a Colombian university assessed attitudes about counseling, personal attitudes, physical activity, personal nutrition, risky alcohol consumption, smoking, inadequate nutrition, and noncompliance; survey results were also compared against the overall university environment where the medical students were studying and showed that despite the university's effort to encourage better nutrition among medical students, inadequate nutrition and sedentary habits continued to increase throughout their medical education [8]. Students who reported more physical activity were more likely to counsel patients on preventive health. Further, these students also reported healthier lifestyle choices, defined by increased fruit and vegetable consumption, less tobacco use, and less alcohol consumption [8].

The National Research Council (NRC), which provides objective advice to the U.S. government relating to science and technology, has established a minimum requirement of 25 hours of classroom nutrition education during the preclinical years of medical school to inform future physicians on healthy eating habits. A previous study suggested some inadequacy in such programs [10]. However, another study [9] from 2008 reported that personal diet improved in $60 \%$ of first-year medical students after a health educational program was implemented. Nova Southeastern University Kiran C. Patel College of Osteopathic Medicine (NSU-KPCOM) and other professional HP programs currently include nutritional education in their respective first-year curriculums. Because HP students tend to receive more nutritional education than non-health professional (NHP) students, the primary goal of this study was to evaluate whether this education creates differences between HP and NHP students' everyday diet and exercise practices.

To evaluate these potential differences, we elected to utilize a survey-based methodology because this approach to collecting vast obesity trend data is utilized by other organizations. Currently, the World Health Organization (WHO) uses self-reported surveys to collect much of its data pertaining to obesity [11]. Self-reporting is known to lead to errors in data collection, yet is the mainstay in understanding the prevalence of obesity worldwide. The CDC uses a variety of survey systems to collect their data regarding obesity. The Behavior Risk Factor Surveillance System (BRFSS) is the largest ongoing telephone survey system and tracks current health conditions, risky behavior, and preventative behavior. Other surveys, such as the National Health and Nutrition Examination Survey (NHANES) and the Youth Risk Behavior
Surveillance System (YRBSS), specifically pertain to nutrition [1]. The YRBSS utilizes a survey for data collection regarding nutritional habits in a specific population.

We hypothesized that students in HP programs would report different diet and exercise habits compared with students in NHP programs.

\section{Methods}

This study was reviewed and determined exempt by the Nova Southeastern University institutional review board (IRB No. 2018-455-NSU).

A 16-question survey was created and distributed to NSU students enrolled in both HP and NHP programs. NHP programs included graduate-level programs in nonmedical fields. Survey invitations were distributed to students of any year throughout their respective program. The survey was distributed to HP programs utilizing an allinclusive university email server, and the survey was distributed to students in NHP programs via an email from the dean of each respective program. The survey was distributed to programs in September of 2018, with one reminder email sent to each program three months after initial distribution. The survey was open for responses until September of 2019. Google Survey (Google, Inc.) was used to create the survey and store response data.

The full survey is available as Supplementary Material. Questions were designed to cover a broad range of dietary and exercise information while remaining simple and concise. The first six questions focused on demographic information including the respondent's sex, age, ethnicity, living situation, dietary preference (omnivore, vegetarian, vegan), and the specific professional program in which the respondent was enrolled. The remaining 10 questions focused on how often the student consumed vegetables, fruit, protein, junk food, sweets (candy, ice cream, cookies, etc.), red meat, water, and caffeine per week, as well as how often the respondent exercised and considered making healthy food choices per week.

Each of the final 10 questions had five answer choices, with answer choice " $\mathrm{A}$ " designated as the minimum value of the question topic being examined, and answer choice "E" as the maximum value. For example, choice " $\mathrm{A}$ " was correlated with the least amount of exercise time/week (0-30 $\mathrm{min} /$ week), and choice "E" with the most (120-150 min/week). Answer choices A-E were then assigned a "code value" that was used to assess results; these "code values" were assigned in either ascending or descending order by $2 \mathrm{~s}(2,4,6,8,10$ or $10,8,6,4,2)$, with the highest value being assigned to the preferred choice. For example, answer choice A was assigned a "code value" of two, answer choice B was assigned a "code value" of four, answer choice $C$ was assigned a "code value" of six, answer choice D was assigned a "code value" of eight, and answer choice E was assigned a code value of 10 when participants were asked how many vegetables they consumed per day, but the converse "code values" were assigned when participants were asked how much red meat they consumed per week, with answer choice A assigned the "code value" of 10 . Of the 10 coded questions, six were devoted to different food categories (high-protein food, red meat, sweets, fast food, fruits, and vegetables), one to fluids (water), one to caffeine intake, one to exercise, and one to self-assessment of diet. 


\section{Analysis of survey data}

Comparisons between groups (HP vs. NHP) were based on the average coded values for each of the 10 coded questions. The analysis of variance statistical method was utilized to analyze survey data. Tests for normality of values were based on the Shapiro-Wilk criteria for each question. Results showed that the normality criteria were not satisfied for any of the questions $(\mathrm{p}<0.001)$. Thus, all comparisons between groups were based on the nonparametric Mann-Whitney $\mathrm{U}$ test. Responses for each of the 10 questions were evaluated separately, and a $\mathrm{p}<0.01$ was considered significant. A similar approach was used for the comparison of responses for men and women; all responses were included in the analysis by sex independent of the program in which the respondent was enrolled.

\section{Results}

Of 732 total respondents, 569 (77.7\%) were HP students and $163(22.3 \%)$ were NHP students; respondents' area$\mathrm{s}$ of study are summarized in Table 1 . The majority (527; 72\%) were women (205 [28\%] men). Self-reported racial and ethnic distribution analysis showed that 371.9 (50.8\%) respondents were White, 167 (22.8\%) were Hispanic, $56(7.7 \%)$ were Black or African-American, 56 (7.6\%) were Asian, 44 (6.0\%) selected Asian-Indian, and $37.3(5.1 \%)$ selected the "other" category from the survey.
Results showed no significant HP-NHP difference ( $p>0.01)$ in any parameter including the consumption of sweets, fast food, red meat, caffeine, water, fruit, and vegetables, as well as healthy choices and exercise. Mean "code values" for each survey question are shown in Table 2 . While there were no statistically-significant differences found between HP and NHP students, there were statistically-significant

Table 2: Mean "code values" for HP and NHP respondents by survey topic.

\begin{tabular}{|c|c|c|c|c|}
\hline & \multicolumn{2}{|c|}{ Program comparison } & \multicolumn{2}{|r|}{ Sex comparison } \\
\hline & HP & NHP & Men & Women \\
\hline we & $30 \pm 1.78$ & $6.41 \pm 1.71$ & $6.82 \pm 1.66$ & $\star \star 6.19$ \\
\hline Fast food & $7.62 \pm 1.82$ & $7.55 \pm 1.85$ & $7.30 \pm 1.92$ & $7.70 \pm 1.80$ \\
\hline ed meat & $7.02 \pm 2.04$ & $6.74 \pm 2.05$ & $5.96 \pm 2.03$ & $\star * 7.34 \pm 1.90$ \\
\hline Caffeine & $4.92 \pm 2.62$ & $5.31 \pm 2.76$ & $5.02 \pm 2.77$ & $5.00 \pm 2.62$ \\
\hline Protein & $4.56 \pm 1.12$ & $4.61 \pm 1.18$ & $4.90 \pm 1.23$ & $\star \star 4.41 \pm 1.03$ \\
\hline Water & $6.45 \pm 1.91$ & $6.64 \pm 2.27$ & $6.85 \pm 1.90$ & $\star \star 6.23 \pm 1.96$ \\
\hline Fruit & $3.96 \pm 1.04$ & $3.79 \pm 0.96$ & $3.80 \pm 1.13$ & $3.96 \pm 0.99$ \\
\hline Vegeta & $4.17 \pm 1.05$ & $4.22 \pm 1.11$ & $4.24 \pm 1.14$ & $4.12 \pm 1.01$ \\
\hline $\begin{array}{l}\text { Healthy } \\
\text { choices }\end{array}$ & $7.69 \pm 1.58$ & $7.51 \pm 1.82$ & $7.54 \pm 1.66$ & $7.68 \pm 1.60$ \\
\hline Exercise & $5.78 \pm 3.01$ & $6.20 \pm 3.09$ & $6.36 \pm 3.01$ & $\star 5.62 \pm 3.00$ \\
\hline
\end{tabular}

${ }^{*} \mathrm{p}<0.01$, statistically significant difference in values between sexes. $\star \star p<0.001$, statistically significant difference in values between sexes. HP, healthcare professional; NHP, non-health professional.

Table 1: Response distribution for students in health professions and non-health professions programs $(n=732)$.

\begin{tabular}{|c|c|c|c|}
\hline \multirow[t]{2}{*}{ Professional programs } & \multicolumn{3}{|r|}{ Respondents } \\
\hline & n (\%) & Men, n (\%) & Women, n (\%) \\
\hline \multicolumn{4}{|l|}{ Health professions } \\
\hline Anesthesiology assistant & $39(5.3)$ & $14(43.8)$ & $25(64.2)$ \\
\hline Dental medicine & $18(2.5)$ & $9(50)$ & $9(50)$ \\
\hline Medical sciences & $17(2.3)$ & $8(47.1)$ & $9(52.9)$ \\
\hline Nursing & $13(1.8)$ & $1(7.7)$ & $12(92.3)$ \\
\hline Occupational therapy & $84(11.5)$ & $9(10.7)$ & $75(89.3)$ \\
\hline Osteopathic medicine & $174(23.8)$ & $66(37.9)$ & $108(62.1)$ \\
\hline Pharmacy & $73(10.0)$ & $12(16.4)$ & $61(83.6)$ \\
\hline Physical therapy & $65(8.9)$ & $24(36.9)$ & $41(63.1)$ \\
\hline Physician assistant & $86(11.7)$ & $17(19.8)$ & $69(80.2)$ \\
\hline Health professions total & $569(77.7)$ & $160(28.1)$ & $409(71.9)$ \\
\hline \multicolumn{4}{|l|}{ Non-health professions } \\
\hline College of Arts, Humanities \& & $2(0.3)$ & $1(50)$ & $1(50)$ \\
\hline \multicolumn{4}{|l|}{ Social Science } \\
\hline College of Business and Entrepreneurship & $79(10.8)$ & $20(25.3)$ & $59(74.7)$ \\
\hline College of Law & $65(8.9)$ & $22(33.8)$ & $43(66.2)$ \\
\hline College of Natural Sciences and Oceanography & $10(1.4)$ & $2(20)$ & $8(80)$ \\
\hline College of Psychology ${ }^{a}$ & $7(1.0)$ & $0(0)$ & $7(100)$ \\
\hline Non-health professions total & $163(22.3)$ & $45(27.6)$ & $118(72.4)$ \\
\hline Grand total & 732 & $205(28.0)$ & $527(72.0)$ \\
\hline
\end{tabular}

${ }^{a}$ College of Psychology was deemed a non-health professions due to the universities specific categorization of programs listed under the Health Professions Divisions title. 
differences between sexes irrespective of the HP and NHP programs in which they were enrolled (Table 2). Women had higher mean "code values" than men in weekly red meat consumption ( $7.34 \pm 1.9$ vs. $5.96 \pm 2.03$; $p<0.001)$, indicating that they consumed it fewer times per week. Women had lower mean "code values" than men in weekly sweet consumption ( $6.19 \pm 1.74$ vs. $6.82 \pm 1.66$; $p<0.001)$, indicating that they consumed it more frequently. Women also had lower mean "code values" for daily water intake $(6.23 \pm 1.96$ vs. $6.85 \pm 1.90 ; \mathrm{p}<0.001)$ and daily protein intake $(4.41 \pm 1.03$ vs. $4.90 \pm 1.23 ; \mathrm{p}<0.001$ ), indicating that they consumed both less frequently. Women also had a lower mean "code value" for minutes of weekly exercise value than men $(5.62 \pm 3.0 \mathrm{vs}$. $6.36 \pm 3.00 ; \mathrm{p}<0.01$ ), indicating that they did not exercise as much.

\section{Discussion}

Our hypothesis prior to administration of the survey was that HP students would demonstrate different nutritional and lifestyle choices than their NHP counterparts. The results showed that for all 10 coded questions regarding diet, lifestyle choices, and exercise, there was no statistically significant difference between HP and NHP students. This indicates that despite differences in program curricula, HP and NHP students demonstrate similar dietary, lifestyle, and exercise habits.

One question worthy of discussion is whether the quantity of nutrition education in HP curricula is sufficient. Among the four HP programs in our study with the highest number of respondents, it is difficult to determine exactly how much nutritional education each student receives. For example, students in the osteopathic medicine program attend a newly implemented 1-credit nutrition course. The physician assistant program offers two credit hours on Complementary Medicine \& Nutrition, as well as three credit hours in Health Promotion and Disease Prevention. The occupational therapy curriculum includes multiple courses regarding mental health and wellness, as well as environment and lifestyle, pertaining to occupational medicine. The physical therapy program contains three credit hours on the Essentials of Exercise Physiology, Health Promotion, and Wellness, along with a Healthcare Educator course for one credit hour. While all these curricula contain dedicated nutritional courses, nutritional education might also be incorporated into other coursework, such as biochemistry. Furthermore, clinical rotations remain a crucial aspect of each program's curriculum. It is difficult to assess the precise amount of nutritional education that HP students receive during their clerkship, because these experiences vary depending on each student's preceptor and clinic location.

It has been reported that only $27 \%$ of medical schools teach the recommended 25 hours of nutritional education [12]. An average of 19.6 hours of nutritional education was incorporated throughout the four years of medical school, which accounts for less than $1 \%$ of total medical lecture hours. It was also found that the majority of this nutritional education involved medical biochemistry, rather than specific nutritional counseling [12]. Data suggest that $71 \%$ of incoming medical students reported that they believe nutrition is clinically important; however, upon graduation, less than half of them maintained this opinion [12]. Beyond medical school, fewer than $14 \%$ of physicians believe that they received appropriate training in nutritional counseling for patients [12]. The lack of emphasis on nutritional education in medical school may be explained by the competition for time with other subjects in the medical curricula [11]. The introduction of new courses, such as those focused on nutritional education, is met with resistance not only because there is limited time available, but also because the appropriate faculty to lead such a course are lacking clinical experience [13]. Another reason for the lack of nutritional education is that there is a greater focus on disease diagnostics and intervention rather than disease prevention [11]. Finally, even if a medical school curriculum incorporates the 25 hours recommended by the NRC, there is no proposed standardized curriculum [11].

The Association of American Medical Colleges (AAMC) released an updated report with updated curriculum recommendations for medical schools in 2017 [14]. These recommendations were classified into three subjects of medical education: basic sciences, clinical sciences, and population health sciences. Basic science pertains to classroom education on the fundamentals of obesity. This includes the physiology of diet and hunger, energy balance, physical activity, and energy consumption. Clinical science involves the practical application of nutritional and dietary education, including topics such as calculating BMI, measuring waist circumference, and understanding the secondary causes of obesity and its comorbidities. Finally, population health science refers to the epidemiology of obesity and its effect on public policy, social factors, and environmental factors that influence a population's lifestyle and nutritional choices $[15,16]$.

Continuity of nutritional education and its incorporation into clinical practice are two specific challenges facing medical students. A systematic review published in 2012 by Vitolins et al. [16] compared separate studies regarding medical student education on obesity intervention training. They found that none of the studies in their review integrated 
obesity education continuously throughout all four years of medical school. They also discovered a lack of published evidence pertaining to medical obesity education, which they believe coincides with physician reports of inadequate training in patient weight management [16-22]. Additionally, they reviewed five different studies that assessed the various methods in which students were educated on the application of nutritional and lifestyle information [16]. One of the studies, a systematic review by Vitolins et al. [16], included a previous study [23] examining 115 first-year medical students and evaluating their response to two interactive lectures and two standardized patient activities that were focused on nutrition and exercise counseling. Of the 57 medical students who completed the pre- and post-intervention questionnaire, there was a reported increase in confidence of nutritional counseling and exercise prescription [16, 22, 23]. Carson [24] examined fourth-year medical students who were provided a tape measure, pocket reference card with nutritional and exercise information, and two computer-based patient cases. A multiple-choice quiz was administered to the students prior to the distribution of the items and then again after their use in a clinical setting. Correct responses regarding the interpretation of waist circumference rose from $82 \%$ (pre-clerkship quiz) to $98.5 \%$ (post-clerkship quiz) [16, 24].

Regarding the results of our study, it is likely that dietary and lifestyle habits may have already been developed prior to the onset of graduate-level education, irrespective of HP or NHP programs. This may explain the reason why no statistically significant difference was found between HP and NHP students. After all, each student who responded to the survey obtained at least an undergraduate degree, with the exception of nursing students who were included in this study because nursing is considered a HP program. As previously mentioned, obesity is less prevalent in college graduates compared to those with a high-school degree or equivalent [4]. A study comparing the prevalence of obesity between graduate and undergraduate students would be of interest and may support this finding, if no statistically significant differences are found between the two groups.

The prevalence of obesity among healthcare professionals is estimated at $22 \%$ [8]. It has been found that physicians who were considered obese provided far less nutritional and exercise information to their patients [19, 25]. As Dietz et al. [26] explained, just as physicians who smoke are less likely to counsel their patients about smoking, a physician's own BMI is indicative of the likelihood that they will counsel their patients who are obese.

If the nutritional education-related curriculum for future healthcare professionals is indeed insufficient as currently constructed, potential solutions should be explored. One logical proposal may be to augment existing medical curricula with an interactive, comprehensive, and free online nutritional education system such as "Nutrition in Medicine" [11]. This online system provides 15-minute modules involving basic nutritional education as well as assessment and intervention learning, thus providing students with preventative and therapeutic aspects of nutrition. This solution would bypass the aforementioned challenges such as the lack of appropriate faculty, resistance to incorporation due to scheduling conflicts and time commitment, and the need for designed objectives [11]. However, even if healthcare curricula were altered to sufficiently deliver nutritional and exercise education, the issue of nutrition and its clinical application would remain. The failure to incorporate nutritional recommendations into clinical practice may not be due to inadequate education during medical school but rather due to the lack of continued nutritional training throughout residency and fellowship. Through their research, Eisenberg and Burgess [12] discovered that the word "nutrition" was never mentioned on Internal Medicine licensing board examinations. Even in cardiology fellowships, nutritional counseling was omitted from the required training [12]. Correction of America's obesity pandemic will require an all-encompassing team effort involving changes made through governmental policy, healthcare reform, improved physician training, and individual patient efforts.

There are potential solutions to the ongoing issues illustrated in our research. In his book Upstream, Dan Heath discusses how healthcare experts (namely primary care physicians) are best equipped to reduce the amount of patient hospitalizations, emergency room visits, and re-admissions [27]. However, primary care physicians overcome with insurmountable pressure to meet patient quotas and abide by insurance regulations - understandably struggle in preventing patient hospitalizations, ER visits, and re-admissions. The Accountable Care Organization (ACO) was implemented in the 2010 Affordable Care Act in an attempt to solve the aforementioned struggles [27-29]. ACOs are created by coordinated, physician-formed groups. Using estimates of expected hospital visits, Medicare calculates the potential annual costs of each patient under an ACO. Thus, through this plan, if physicians were to reduce the amount of hospitalizations among their patients, the money that Medicare saved would be shared with the participating physicians. This created an incentive for physicians, who started spending more time with each patient and focused more on patients' blood sugar levels, blood pressure, diet, and exercise [27]. Their focus shifted from reacting to a patient who was already hospital-bound to prevention through adequate nutritional and lifestyle education [27]. 
The ACO was estimated to reduce combined patient costs $\$ 470$ million from 2012 to 2015 [28]. It should be noted that seven of the 32 pioneer ACOs withdrew from the program because no savings were demonstrated in 2012 [29]. Although the perfect plan does not exist, this innovative thinking provides promise for possible future solutions.

There are limitations to this study. Survey results are often inaccurate and experience response bias. Also, considering that women accounted for $72.6 \%$ of survey responses, this justly could be regarded as a limitation when comparing the results of this study between men and women.

\section{Conclusions}

The main result of this survey-based research demonstrated that there is no difference in dietary and exercise practices between HP and NHP students. A lack of emphasis on nutritional education in HP settings, especially medical school, is a possible reason for the lack of difference in dietary habits among HP and NHP students. Low physician competency regarding nutritional education results in abandonment of nutritional assessment and counseling for their patients [11]. It is therefore important to provide future medical professionals adequate nutritional training. Future studies should investigate not only how to improve nutritional education among potential medical professionals, but also how to develop methods that will allow them to translate this understanding into their future practice.

Research funding: None reported.

Author contributions: All authors provided substantial contributions to conception and design, acquisition of data, and analysis and interpretation of data; all authors drafted the article or revised it critically for important intellectual content; all authors gave final approval of the version of the article to be published; and all authors agree to be accountable for all aspects of the work in ensuring that questions related to the accuracy or integrity of any part of the work are appropriately investigated and resolved.

Competing interests: Authors state no conflict of interest. Informed consent: Informed consent was not obtained. Ethical approval: This study was reviewed and determined exempt by the Institutional Review Board at Nova Southeastern University (IRB No. 2018-455-NSU).

\section{References}

1. Centers for Disease Control and prevention. Surveillance systems. Available from: https://www.cdc.gov/obesity/data/ surveillance.html [Accessed 25 Jul 2020].
2. Katherine M, Flegal PD. Trends in obesity among Adults in the United States, 2005 to 2014. Available from: https:// jamanetwork.com/journals/jama/fullarticle/2526639 [Accessed 25 Jul 2020].

3. Menezes MCD, Duarte CK, Costa DVDP, Lopes MS, de Freitas PP, Campos SF, et al. A systematic review of effects, potentialities, and limitations of nutritional interventions aimed at managing obesity in primary and secondary health care. Nutrition 2020;7576:110784.

4. Centers for Disease Control and Prevention. Adult obesity prevalence maps. Available from: https://www.cdc.gov/obesity/ data/prevalence-maps.html [Accessed 25 Jul 2020].

5. World Health Organization. Obesity and overweight. Available from: https://www.who.int/news-room/fact-sheets/detail/ obesity-and-overweight [Accessed 25 Jul 2020].

6. Information for CME Credit. Prevalence of obesity among U.S. Workers and associations with occupational factors. Am J Prev Med. 2014;46:237-48.

7. Miller SK, Alpert PT, Cross CL. Overweight and obesity in nurses, advanced practice nurses, and nurse educators. J Am Acad Nurse Pract 2008;20:259-65.

8. Alba LH, Badoui N, Gil F. Attitude toward preventive counseling and healthy practices among medical students at a Colombian university. Front Med 2015;9:251-9.

9. Barss P, Grivna M, Al-Maskari F, Kershaw G. Strengthening public health medicine training for medical students: development and evaluation of a lifestyle curriculum. Med Teach 2008;30:e196218.

10. Adams KM, Kohlmeier M, Powell M, Zeisel SH. Nutrition in medicine. Nutr Clin Pract 2010;25:471-80.

11. Bauman A, Rutter H, Baur L. Too little, too slowly: international perspectives on childhood obesity. Publ Health Res Pract 2019; 29:2911901.

12. Eisenberg DM, Burgess JD. Nutrition education in an era of global obesity and diabetes. Acad Med 2015;90:854-60.

13. Kris-Etherton PM, Akabas SR, Bales CW, Bistrian B, Braun L, Edwards MS, et al. The need to advance nutrition education in the training of health care professionals and recommended research to evaluate implementation and effectiveness. Am J Clin Nutr 2014;99:1153S-66S.

14. Maeshiro R. Contemporary issues in medicine: the prevention and treatment of overweight and obesity. Association of American Medical Colleges; 2007. Available from: https://store. aamc.org/downloadable/download/sample/sample_id/57/.

15. BSE. Expert committee recommendations regarding the prevention, assessment, and treatment of child and adolescent overweight and obesity: summary report. Available from: https:// pubmed.ncbi.nlm.nih.gov/18055651/ [Accessed 25 Jul 2020].

16. Vitolins MZ, Crandall S, Miller D, Ip E, Marion G, Spangler JG. Obesity educational interventions in U.S. Medical schools: a systematic review and identified gaps. Teach Learn Med 2012;24:267-72.

17. Kolasa K. Developments and challenges in family practice nutrition education for residents and practicing physicians: an overview of the North American experience. Eur J Clin Nutr 1999; 53:589-96.

18. Beno L, Hinchman J, Kibbe D, Trowbridge F. Design and implementation of training to improve management of pediatric overweight. J Continuing Educ Health Prof 2005;25:248-58.

19. Kopelman P. Developing an action plan for obesity education. Int J Obes 2001;25:S16-9. 
20. Bruer RA, Schmidt RE, Davis H. Commentary: nutrition counseling -should physicians guide their patients? Am J Prev Med 1994;10: 308-11.

21. Walsh CO, Ziniel SI, Delichatsios HK, Ludwig DS. Nutrition attitudes and knowledge in medical students after completion of an integrated nutrition curriculum compared to a dedicated nutrition curriculum: a quasi-experimental study. BMC Med Educ 2011;11:58.

22. Block JP, Desalvo KB, Fisher WP. Are physicians equipped to address the obesity epidemic? Knowledge and attitudes of internal medicine residentsH. Prev Med 2003;36:669-75.

23. lii PFB, Stetson BA, Rising W, Wesley GC, Ritchie CS. Development and evaluation of a nutrition and physical activity counseling module for first-year medical students. Med Educ Online 2004;9: 4359.

24. Carson JAS. Pocket tape measure for waist circumference: training medical students and residents on a simple assessment of body composition. J Nutr 2003;133:547S-9S.

25. Tong EK, Strouse R, Hall J, Kovac M, Schroeder SA. National survey of U.S. health professionals' smoking prevalence, cessation practices, and beliefs. Nicotine Tob Res 2010;12: 724-33.

26. Dietz WH, Baur LA, Hall K, Puhl RM, Taveras EM, Uauy R, et al. Management of obesity: improvement of health-care training and systems for prevention and care. Lancet 2015;385: 2521-33.

27. Heath D. Upstream: the quest to solve problems before they happen. New York: Avid Reader Press; 2020.

28. Federal Register. Medicare Program; Medicare shared savings program; accountable care organizations-pathways to success. Available from: https://www.federalregister.gov/documents/ 2018/08/17/2018-17101/medicare-program-medicare-sharedsavings-program-accountable-care-organizations-pathways-tosuccess [Accessed 25 Jul 2020].

29. Mcwilliams JM. Changes in Medicare shared savings program savings from 2013 to 2014. Jama 2016;316:1711.

Supplementary Material: The online version of this article offers supplementary material (https://doi.org/10.1515/jom-2020-0178). 